\title{
Deuterium Residual Quadrupolar Couplings: Crossing the Current Frontiers in the Relative Configuration Analysis of Natural Products
}

\author{
Philippe Lesot, ${ }^{\mathrm{a}, \mathrm{b}, *}$ Roberto R. Gil, ${ }^{\mathrm{c}, *}$ Philippe Berdagué, ${ }^{\mathrm{a}}$ and Armando Navarro-Vázquez ${ }^{\mathrm{d}}$ \\ ${ }^{\text {a } U n i v e r s i t e ́ ~ P a r i s-S a c l a y, ~ U F R ~ d ' O r s a y, ~ R M N ~ e n ~ M i l i e u ~ O r i e n t e ́, ~ I C M M O, ~ U M R ~ C N R S ~ 8182, ~ B a ̂ t . ~ 410, ~} 15$ rue du \\ Doyen, Georges Poitou, F-91405 Orsay cedex, France. \\ ${ }^{\mathrm{b}}$ Centre National de la Recherche Scientifique (CNRS), 3 rue Michel Ange, F-75016 Paris, France. \\ ${ }^{c}$ Department of Chemistry, Carnegie Mellon University, Pittsburgh, PA, USA.

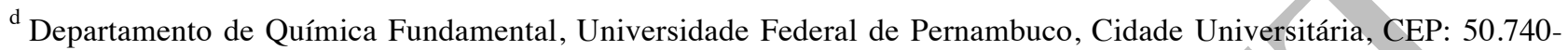 \\ 540 Recife, PE, Brazil.
}

\begin{abstract}
:
Determination of the 3D structure (configuration and preferred conformation) of complex natural and synthetic organic molecules is a long-standing but still challenging task for chemists, with various implications in pharmaceutical sciences whether or not these substances have specific bioactivities. NMR in aligning media, either lyotropic liquid crystals (LLC) or polymer gels, in combination with molecular modelling is a unique framework to solve complex structural problems whose analytical wealth lies in the establishment of non-local structural correlations. As an alternative to already well-established anisotropic NMR parameters, such as RDCs (residual dipolar couplings) and RCSAs (residual chemical shift anisotropies), it is shown here that deuterium residual quadrupolar couplings $\left({ }^{2} \mathrm{H}-\mathrm{RQCs}\right)$ can be extracted from ${ }^{2} \mathrm{H}$ 2D-NMR spectra recorded at natural abundance level in samples oriented in a homopolypeptide LLC (PBLG). These ${ }^{2} \mathrm{H}$-RQCs were successfully used to address non-trivial structural problems in organic molecules. The performance and scope of this new tool is examined for two natural chiral compounds of pharmaceutical interest (strychnine and artemisinin). This is the first report in which the 3D structure/relative configuration of complex bioactive molecules is unambiguously determined using only ${ }^{2} \mathrm{H}$-RQCs, being in this case at ${ }^{2} \mathrm{H}$ natural abundance.
\end{abstract}

Keywords: 3D molecular structure, Relative configuration, Natural bioactive products, Deuterium, Natural abundance, 2D-NMR, Alignment tensor, Anisotropic solvent, PBLG

Corresponding authors: philippe.lesot@universite-paris-saclay.fr rgil@andrew.cmu.edu

Supporting information: Supporting information available

ORCID numbers ${ }^{\bullet}$ : - Philippe Lesot : orcid.org/0000-0002-5811-7530

- Roberto R. Gil : orcid.org/0000-0002-8810-5047

- Armando Navarro-Vázquez : orcid.org/0000-0003-4364-516X 


\section{INTRODUCTION}

Structure elucidation, including configurational/conformational analysis of complex mole-cules of natural or synthetic origin is a fascinating domains of molecular sciences, but also an indispensable "investigation game" for chemists, thus offering a unique way to reveal the originality, the complexity and wealth of molecular structures in Nature. ${ }^{1-5}$ Concomitantly, this knowledge and the numerous associated implications (spatial structure, conformational dynamics, binding-site geometry, etc.) are crucial in the analysis of molecular bioactivity. ${ }^{6,7}$ Exploring new opportunities and promoting pioneering alternatives to existing tools while expending the arsenal of analytical possibilities deserves therefore a very special attention.

The determination of relative configuration in organic compounds is still a challenging problem since single crystal X-ray diffraction spectroscopy is in many cases not applicable due to the studied compound failing to produce suitable crystals for analysis. Since powder X-ray ${ }^{8,9}$ diffraction analysis, or the very promising molecular sponge ${ }^{10}$ methods still present important technical issues, liquid-state NMR spectroscopy ${ }^{11}$ assisted by molecular modeling, becomes the technique of choice for probing molecular geometry. Classically, the problem of obtaining internuclear distances and bond angles is addressed through the analysis of nuclear Overhauser effect (NOE) experiments ${ }^{12,13}$ as well as the determination of homonuclear ${ }^{3} J\left({ }^{1} \mathrm{H}-{ }^{1} \mathrm{H}\right)$ and/or heteronuclear ${ }^{1} J\left({ }^{1} \mathrm{H}^{-13} \mathrm{C}\right)$ scalar couplings. ${ }^{14,15}$ New developments in quantitative-NOE methods ${ }^{16}$ or DFT prediction of chemical shifts/scalar couplings ${ }^{17-19}$ further enhance the power of these approaches. However, assignment of relative configuration can still be a challenging problem when stereogenic centers are remotely located in the molecule, and hence magnetically disconnected. ${ }^{9}$ The advent of NMR in weakly aligning media (chiral or not) allowed made it possible to overcome these hurdles by recovering a small fraction of the orientational (tensorial) information inherent to NMR observables, and therefore establishing angular restrictions on the relative orientation of the associated tensors. ${ }^{20}$ Most of the existing examples, some of them involving difficult structural problems, are based on 
the use of one-bond proton-carbon residual dipolar couplings $\left(\left({ }^{13} \mathrm{C}-{ }^{1} \mathrm{H}\right)-\mathrm{RDC}\right)$, noted also as ${ }^{1} D_{\mathrm{CH}} \cdot{ }^{21,22}$ These key heteronuclear dipolar data can be also soundly combined with: (i) long-range ${ }^{2,3} D_{\mathrm{CH}}$ constant analysis, ${ }^{23-25}$ (ii) NOE constraints, ${ }^{22,25}$ (iii) ${ }^{3} J_{\mathrm{HH}}$ data, ${ }^{21,22,26,27}$ or (iv) even powder Xray diffraction analysis. ${ }^{9}$ Moreover, information on relative orientation of non-protonated carbons can be obtained through the use of the ${ }^{13} \mathrm{C}$ residual chemical shift anisotropy $\left({ }^{13} \mathrm{C}-\mathrm{RCSA}\right) \cdot{ }^{28-33}$ Although ${ }^{13} \mathrm{C}$-RCSAs are much more technically demanding to measure with enough accuracy than RDCs (in particular when very weakly oriented media are used) the associated experimental protocol is somewhat simplified as only ${ }^{13} \mathrm{C}-\left\{{ }^{1} \mathrm{H}\right\}$ 1D NMR spectra need to be measured. ${ }^{34}$

(a)

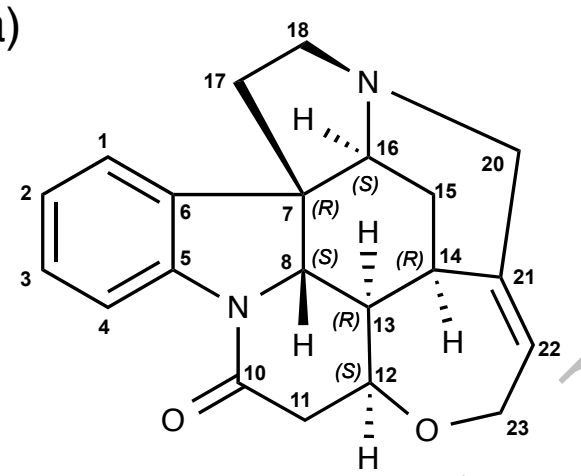

(b)

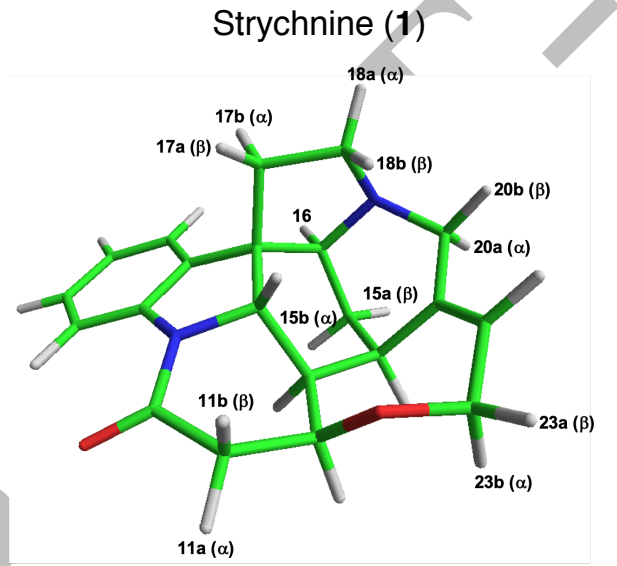

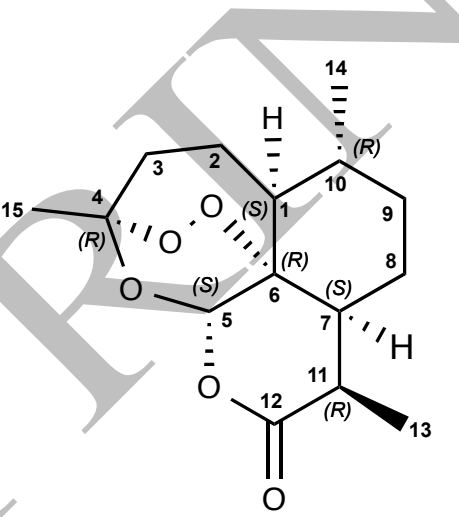

Artemisinin (2)

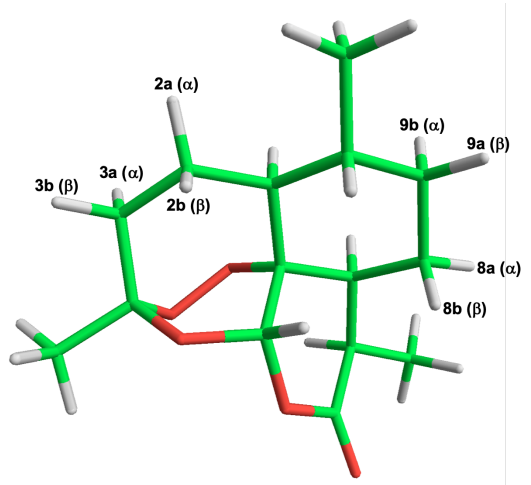

Figure 1. (a) Molecular structures of strychnine (1) and artemisinin (2) along with atomic labels and the corresponding $R / S$ descriptors indicating their absolute configurations: $7 R, 8 S, 12 S, 13 R, 14 R, 16 S$ and $1 S$, $4 R, 5 S, 6 R, 7 S, 10 R, 11 R$, respectively. (b) DFT-optimized 3D structures labeling the $\alpha / \beta(\mathrm{a} / \mathrm{b})$ positions of diastereotopic protons/deuterons.

Residual quadrupolar coupling (RQC) is another important anisotropic NMR parameter that has barely paved its avenue into the structure elucidation field. ${ }^{35}$ RQCs are only detectable for nuclei with spin quantum number $I>1 / 2$. 
The lightest quadrupolar atom is deuterium $\left({ }^{2} \mathrm{H}\right)$ with a nuclear spin of $I=1$ and present in any organic molecule at a natural abundance of $\sim 1.55 \times 10^{-2} \%$ (V-SMOW value) relative to proton. Deuterium possesses a small quadrupolar coupling constant $\left({ }^{2} \mathrm{H}-\mathrm{QCC}\right), C_{\mathrm{Q}}$, which makes the $\mathrm{T}_{2}\left({ }^{2} \mathrm{H}\right)$ relaxation times long enough to obtain isotropic or anisotropic ${ }^{2} \mathrm{H}$ spectra with narrow linewidths. ${ }^{35-39}$ At first glance, the notably low sensitivity of the technique (relative receptivity ${ }^{1} \mathrm{H} /{ }^{2} \mathrm{H}$ is $1.7 \times 10^{6}: 1$ ) caused by the low natural abundance of deuterium (NAD) and its poor magnetogyric ratio $\left.\left(\gamma^{1}{ }_{\mathrm{H}}\right) /\left(\gamma^{2}{ }_{\mathrm{H}}\right)=6.515\right)$ appears as primary drawbacks. ${ }^{40,41}$ However, in modern NMR instruments operating at high magnetic fields and equipped with modern electronics and, particularly, cryogenically cooled ${ }^{2} \mathrm{H}$ probes, ${ }^{42,43}$ it is possible to collect $1 \mathrm{D}$ NAD- $\left\{{ }^{1} \mathrm{H}\right\}$ spectra (and even 2D) for samples at moderate concentrations $\left(\sim 10^{-4}-10^{-5}\right.$ mol with $\left.\mathrm{MW} \sim 200-400\right)$, leading to a wide range of analytical applications. ${ }^{44-47}$ In terms of structural elucidation purposes, $\mathrm{NAD}-\left\{{ }^{1} \mathrm{H}\right\}$ NMR in anisotropic media provides nearly equivalent structural information as the one obtained from ${ }^{1} D_{\mathrm{CH}}$ couplings. ${ }^{35}$ However, ${ }^{2} \mathrm{H}-\mathrm{RQCs}$ present important inherent advantages, but also some drawbacks, with respect to ${ }^{1} D_{\mathrm{CH}}$-based methods. On the pro list, the values of ${ }^{2} \mathrm{H}-\mathrm{RQCs}$ are larger than $\left({ }^{13} \mathrm{C}-{ }^{1} \mathrm{H}\right)$-RDCs by nearly one order (sometimes two) of magnitude, dramatically improving the obtainable accuracy in the determination of the molecular alignment tensor. ${ }^{35}$ Interestingly as ${ }^{2} \mathrm{H}-\left\{{ }^{1} \mathrm{H}\right\}$ NMR spectra can be recorded at the natural abundance level, no isotopic enrichment is necessary. ${ }^{39-42}$ Moreover, because all monodeuterated isotopomers for a given molecule form independent dilute ${ }^{2} \mathrm{H}$-spin systems, anisotropic NAD (denoted herein ANAD) measurements are not affected by all the cumbersome strong ${ }^{1} \mathrm{H}-{ }^{1} \mathrm{H}$ coupling effects that frequently hamper accurate determination of ${ }^{1} D_{\mathrm{CH}}$ couplings in $\mathrm{CH}_{2}$ groups. ${ }^{48,49}$

Disregarding the obvious problem with sensitivity, NAD NMR determination also has some additional disadvantages: first, the experimental determination of the sign of the RQCs is not straightforward since there is no isotropic component involved in the ${ }^{2} \mathrm{H}$ splittings $\left(\Delta v_{\mathrm{Q}}\left({ }^{2} \mathrm{H}\right)\right)$, measured in ANAD spectra, contrarily to the case of ${ }^{13} \mathrm{C}$-RCSAs $\left(\Delta \sigma\left({ }^{13} \mathrm{C}\right)\right)$ or the ${ }^{13} \mathrm{C}-{ }^{1} \mathrm{H}$ total 
spin-spin coupling ${ }^{1} T_{\mathrm{CH}},\left({ }^{1} T_{\mathrm{CH}}={ }^{1} J_{\mathrm{CH}}+2^{1} D_{\mathrm{CH}}\right)$, from which the sign of RCSAs or RDCs can be experimentally determined with accuracy. ${ }^{28}$ In fact, this problem can be solved by roughly determining the ${ }^{1} D_{\mathrm{CH}}$ values on the same sample (compared to ${ }^{1} J_{\mathrm{CH}}$ values), the sign of $\Delta v_{\mathrm{Q}}\left({ }^{2} \mathrm{H}\right)$ being opposite to the one corresponding of the ${ }^{1} D_{\mathrm{CH}}$ coupling of the same bond $\left({ }^{13} \mathrm{C}-{ }^{1} \mathrm{H}\right.$ and ${ }^{13} \mathrm{C}$ ${ }^{2} \mathrm{H}$ internuclear vectors are collinear) with the ratio $\left[\Delta v_{\mathrm{Q}}\left({ }^{2} \mathrm{H}\right)\right] /\left[{ }^{1} D_{\mathrm{CH}}\right]$ varying between -11 and -12 (Figure SI-2), as previously described in literature. ${ }^{35}$ Also, the overlapping and interweaving of spectral lines in 1D experiments can make it difficult if not impossible to extract the different residual dipolar or quadrupolar couplings, so it is necessary to resort to $2 \mathrm{D}$ experiments for measuring that data, usually ${ }^{1} \mathrm{H}-{ }^{13} \mathrm{C}$ HSQC type spectra $\left({ }^{13} \mathrm{C}-{ }^{1} \mathrm{H}\right)$-RDCs and QUOSY type 2D experiments for ${ }^{2} \mathrm{H}$-RQCs. ${ }^{50,51,52}$ Once the magnitude of ${ }^{2} \mathrm{H}-\mathrm{RQCs}$ and their signs are determined, the protocol for relative configuration determination is basically identical to that applied to the analysis of RDCs. $^{35,53}$

For each particular configuration an ensemble of conformations is generated through standard procedures. ${ }^{54,55}$ Depending on the particular structure, DFT refinement of molecular geometries can then be applied, if desired, to the ensemble. For each optimized geometry, the electric field gradient tensors at the nuclei positions are computed at the DFT level. Although the difference of ${ }^{2} \mathrm{H}-\mathrm{QCC}$ values between nuclei attached to carbons of the same hybridization are small, it is advisable to compute the electric field gradient (EFG) tensor for each particular conformation in order to have a more general procedure that could potentially handle all kind of situations. ${ }^{35}$ Note however that in the presence of prostereogenic centers, methylene protons (or deuterons) become diastereotopic, giving rise to two different NMR observables. To avoid the need for methylene diastereotopic site assignment, the SVD fitting procedure was performed using the average ${ }^{2} \mathrm{H}$ RQC value for each pair of diastereotopic deuterons in methylene groups. This is a well-known protocol successfully used to fit RDCs, which avoids the difficult assignment step. ${ }^{54,55}$ 
We will show here that it is possible to use NAD 2D NMR in polypeptide LLC to record a sufficiently large set of accurate ${ }^{2} \mathrm{H}-\mathrm{RQCs}$, which allows determination of not only the relative configuration (stereogenic centers) of complex chiral organic molecules but also the assignment of their prostereogenic methylene directions $(\alpha, \beta)$. As a proof of concept, we have chosen two complex, chiral, polycyclic natural products (Figure 1). The first is strychnine (1), an alkaloidtype poison but a "beloved" model compound for NMR spectroscopists over decades, ${ }^{56-61}$ the second one is artemisinin (2), an antimalarial drug used against Plasmodium falciparum malaria..$^{27,62-66}$ Throughout this work we will apply the MSpin-RQC computational protocol recently described (Figures SI-1 and SI-2). ${ }^{35}$

\section{RESULTS AND DISCUSSION}

Detection of ${ }^{2} \mathrm{H}$-Quadrupolar Doublets (QDs). The analyzed chiral compounds possess several stereogenic centers and therefore non-equivalent diastereotopic positions associated with each prochiral methylene group (6 for 1 and 4 for 2). Here, the number of theoretically expected QDs to be observed in NAD NMR experiments is 22 and 16 for $\mathbf{1}$ and 2, respectively. A typical example of a tilted/symmetrized NAD 2D map (Q-resolved-type $)^{39,51}$ recorded in the $\mathrm{PBLG} / \mathrm{CHCl}_{3}$ system where analyte and solvent NAD signals do not interfere is shown in Figure 2b (artemisinin (2)). For this particular example, the magnitude of ${ }^{2} \mathrm{H}-\mathrm{RQCs}$ varies in a range of 40-760 Hz, but values up to $1 \mathrm{kHz}$ were measured for strychnine 1 . While the mass fractions of polymer used are standard for PBLG LLC samples (10-15 w/w\%), the ${ }^{2} \mathrm{H}-\mathrm{RQC}$ values measured for these complex structures are globally larger than for smaller rigid molecules, no doubt due to a slower molecular dynamic potentially originating from stronger interactions with the polypeptide fibers. ${ }^{33,67}$

Experimentally, we were able to detect and exploit 20 (/22) and 15 (/16) QDs for 1 and 2, respectively; and their associated ${ }^{2} \mathrm{H}-\mathrm{RQCs}$ (with sign) for the structure elucidation. The absence 
of QDs for sites $\mathrm{H}_{4}$ and $\mathrm{H}_{13(a)}$ in $\mathbf{1}$ and $\mathrm{H}_{5(\text { () }}$ in $\mathbf{2}$ (Table SI-1 and SI-4), while the associated ${ }^{1} \mathrm{H}$ signals are observed in their corresponding ${ }^{1} \mathrm{H}$ 1D-NMR spectra in liquid phases can be explained as follows: (i) the most probable reason is an important deuterium depletion effect (associated with a low ${ }^{2} \mathrm{H} /{ }^{1} \mathrm{H}$ isotopic ratio), leading to a drastic reduction of the QD intensity for a given site; this effect is associated with site-specific isotope fractionation observed in natural compounds (enzymatic synthesis); ${ }^{44-47}$ (ii) excessive ${ }^{2} \mathrm{H}$-line broadening due to short ${ }^{2} \mathrm{H}$ transversal relaxation times $\left(\mathrm{T}_{2}{ }^{*}\right)$ caused by the higher viscosity of the PBLG medium compared to isotropic solutions; (iii) a recycling delay $\left(\mathrm{T}_{R}\right)$ of NAD 2D experiments too short leading to loss of magnetization for ${ }^{2} \mathrm{H}$ sites exhibiting exceptionally long $\mathrm{T}_{1}$ values (less probable). The worst-case scenario arises when the first effect combines with ii) or iii). Recording ANAD 1D/2D spectra with larger analyte amounts or at higher magnetic fields could be an option to improve the detection of these QDs. However, the lack of some ${ }^{2} \mathrm{H}-\mathrm{RQCs}$ does not pre-empt the accomplishment of the structural analysis, if a sufficient number of anisotropic data is still experimentally available.

Strychnine. To evaluate the potential of the analytical contributions of ANAD NMR to the structural analysis of natural products, we first investigated strychnine (1) (Figure 1a). This heptacyclic structure (six stereogenic carbon atoms and six prochiral $\mathrm{CH}_{2}$ groups) has been used as "model" molecule over decades and has been investigated either by theoretical approaches, ${ }^{57}$ isotropic $\mathrm{NMR}^{56,68}$ as well as in oriented media using RDCs, ${ }^{30,58,59}$ or RCSAs, ${ }^{29,30}$ as anisotropic data. Residual dipolar couplings $\left({ }^{1} D_{\mathrm{CH}}\right)$ for 1 were recorded in PBLG and PELG polypeptide. ${ }^{58,69}$ The analysis of the NAD 2D-NMR spectrum of 1 recorded in $\mathrm{PBLG} / \mathrm{CHCl}_{3}$ (Figure 2a) leads to the confident assignment of 20 QDs out of a total of 22 , and thereby 20 signed ${ }^{2} \mathrm{H}-\mathrm{RQCs}$ (Table SI-1) for spectral data. In a first step, these data were used as input for the MSpin-RQC program, along with the MMFF94 force-field computed geometries and DFT-determined EFG tensors $^{35}$ for all different relative configurations (see also the Experimental Section). To simplify 

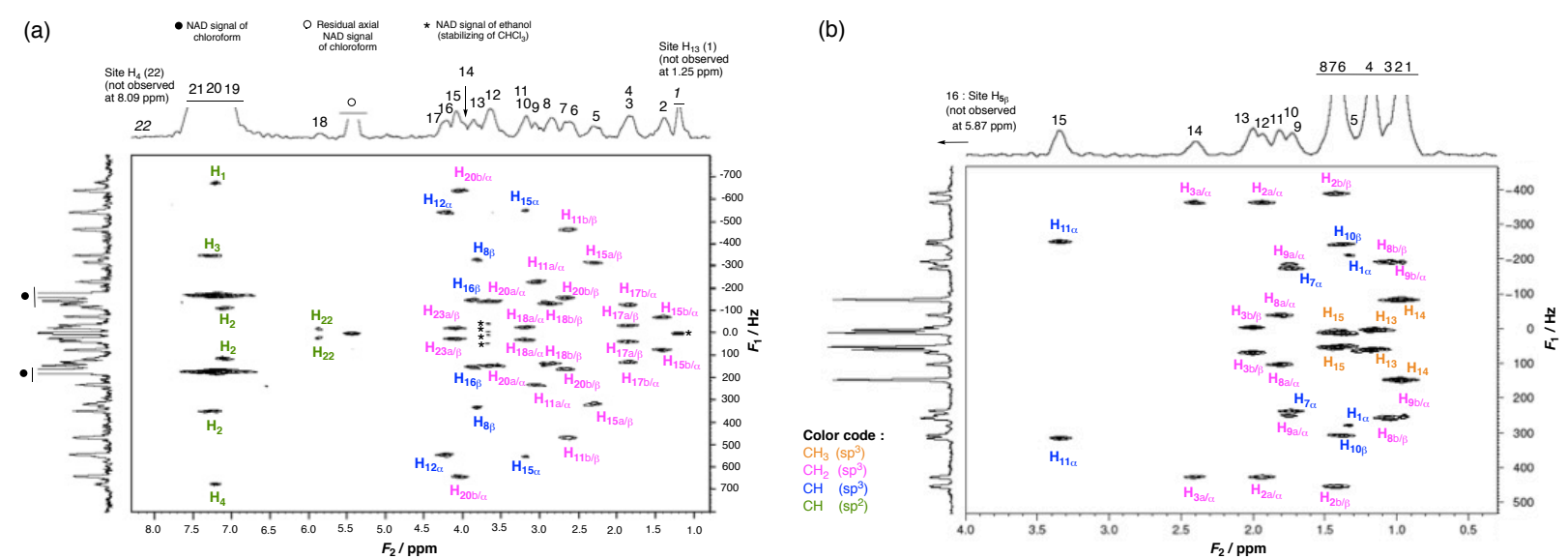

Figure 2. a and b) NAD- $\left\{{ }^{1} \mathrm{H}\right\} Q$-resolved Fz 2D experiments (tilted and then symmetrized maps) of 1 and 2 recorded in $\mathrm{PBLG} / \mathrm{CHCl}_{3}$ phase, along with their ${ }^{2} \mathrm{H}$ quadrupolar doublet assignments according to the DFT (MSpin-RQC) atom labels (Figure 1). ${ }^{2} \mathrm{H}$ chemical shifts (in ppm) and ${ }^{2} \mathrm{H}-\mathrm{RQCs}$ (in $\mathrm{Hz}$ ) of each QD can be directly read from the $F_{2}$ and $F_{1}$ axes, respectively. NAD signal of chloroform is not shown on the map of 2. Larger maps are shown in the Supporting Information.

the naming of each diastereoisomer, the stereochemical descriptors $(R / S)$ were used after the configuration of carbons C-7, C-8, C-12, C-13, C-14 and C16; the correct absolute configuration of strychnine (1) is $7 R, 8 S, 12 S, 13 R, 14 R, 16 S$ (Figure 1a), consequently labeled as $R S S R R S$.

As mentioned above, in a first step allowing to circumvent the necessity of the assignment of two deuterons of methylenes, ${ }^{58}$ the corresponding RQC values were averaged during the fitting. ${ }^{54,55}$ From the conformational search, only two diastereoisomers were identified with more than one conformer within an energy window of $5 \mathrm{kcal} / \mathrm{mol}(R S S R R S$ and $R S R R S R)$. However, for these two configurations, only the lowest energy conformers were used in the fitting process because their populations were energetically favored on more than $99 \%$. The SVD fitting procedure using the RQC average value for the diastereotopic deuterons yielded an excellent degree of discrimination for the correct structure of $\mathbf{1}(7 R, 8 S, 12 S, 13 R, 14 R, 16 S)$ with a Cornilescu's quality $Q$ factor of 0.061 (see Supporting Information for the $Q$ definition). The second-best $Q$ value $(0.118)$ corresponds to the relative configuration $7 R, 8 S, 12 R, 13 R, 14 R, 16 S$. All other configurations lead to significantly higher $Q$ values (Table SI-3).

Table 1 lists all ordering-related parameters obtained from the experimental ${ }^{2} \mathrm{H}-\mathrm{RQCs}$ of $\mathbf{1}$, along with the SVD condition number and quality factor, $Q$. The configuration obtained by the 
RQC protocol is fully consistent with previous reports of $\mathbf{1}$ using $\mathrm{RDCs}^{59,69}$ and $\mathrm{RCSAs}^{30}$ as anisotropic data, as well as with X-ray structure ${ }^{70}$, consequently validating the suitability and the robustness of the ANAD-NMR methodology.

Although the low $Q$ factor obtained for $\mathbf{1}$ indicates a good fit and validates the most probable molecular configuration, the quality of fit between the experimental ${ }^{2} \mathrm{H}-\mathrm{RQC}\left(\mathrm{RQC}{ }^{\text {Exptl }}\right.$ ) and computed back-calculated ${ }^{2} \mathrm{H}-\mathrm{RQCs}\left(\mathrm{RQC}^{\mathrm{Comp}}\right.$ ) can be also visually evaluated from the correlation plot (Figure SI-6). Given the complexity of these structures and the amount of deuterium information, it is not expected that all back-calculated ${ }^{2} \mathrm{H}-\mathrm{NMR}$ based information $\left({ }^{2} \mathrm{H}-\mathrm{RQC} \mathrm{C}^{\mathrm{Comp}}\right)$ values fall within the experimental errors (Table SI-2). In particular, several (combined) effects can lead to the largest of R.D.s, such as structural noise from geometry optimizations, complex vibrational effects, DFT calculations of EFG's, etc.). However, as the final goal is to select the correct configuration, only the magnitude of the quality factor $Q$ (global scoring) must be regarded as the key structure selection criterium.

One of the principal criteria of using ${ }^{2} \mathrm{H}$ anisotropic data over ${ }^{1} D_{\mathrm{CH}}$ is its higher accuracy. Due not only to ${ }^{2} \mathrm{H}$-RQCs being nearly one order of magnitude higher than the corresponding $\left({ }^{13} \mathrm{C}-{ }^{1} \mathrm{H}\right)$ RDCs $\left({ }^{1} D_{C H}\right)$, but also to the fact that each deuterium isotopomer furnishes a pure-shift ${ }^{2} \mathrm{H}$ spectrum (the isotropic NAD- $\left\{{ }^{1} \mathrm{H}\right\}$ 1D spectrum or the $F_{2}$ projection of the ANAD- $\left\{{ }^{1} \mathrm{H}\right\}$ tilted $Q-$ resolved $\mathrm{Fz}$ 2D experiment). This circumvents problems with strong coupling in methylene groups. $^{49}$ Once the correct structure is selected, the diastereotopic deuterons can be straightforwardly assigned in in MSpin-RQC. The program is instructed to perform the fitting to all of the possible combinations of permuted assignments for each prochiral methylene group. The number of possible combinations is equal to $2^{\mathrm{N}}$, when $\mathrm{N}$ is the number of prochiral methylene groups. Strychnine has six prochiral methylene groups, hence a total 64 combinations of permuted assignments were used to find the correct assignment. This combination number drops to 16 (four 
prochiral methylene groups) for artemisinin. The correct assignment for strychnine (1), as reported in the literature, yielded the lowest $Q$ factor of 0.061 .

Finally, all the above fitting procedures were repeated using the correct configuration structure $(7 R, 8 S, 12 S, 13 R, 14 R, 16 S)$, but with a DFT-optimized geometry. Using permutations, a $Q=$ 0.078 was obtained, while a $Q=0.044$ was observed when the average value of RQCs for diastereotopic sites was used. Remarkably, the correlation plot $\left(\mathrm{RQC}^{\mathrm{Comp}} v s\right.$. $\left.\mathrm{RQC} \mathrm{C}^{\mathrm{Exptl}}\right)$ for this structure clearly shows the quality of the fit (Figures SI-7).

As can be seen, no significant differences in $Q$ factors were observed between molecular mechanics and DFT-optimized structure geometries.

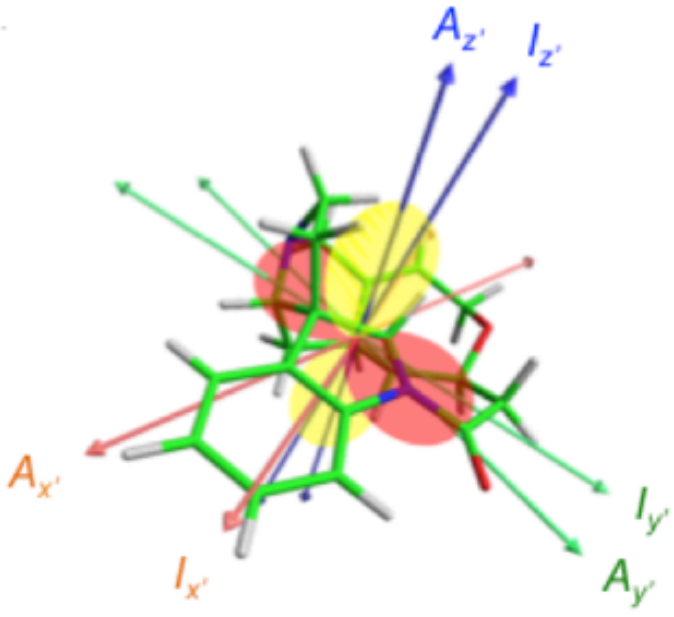

Strychnine (1)

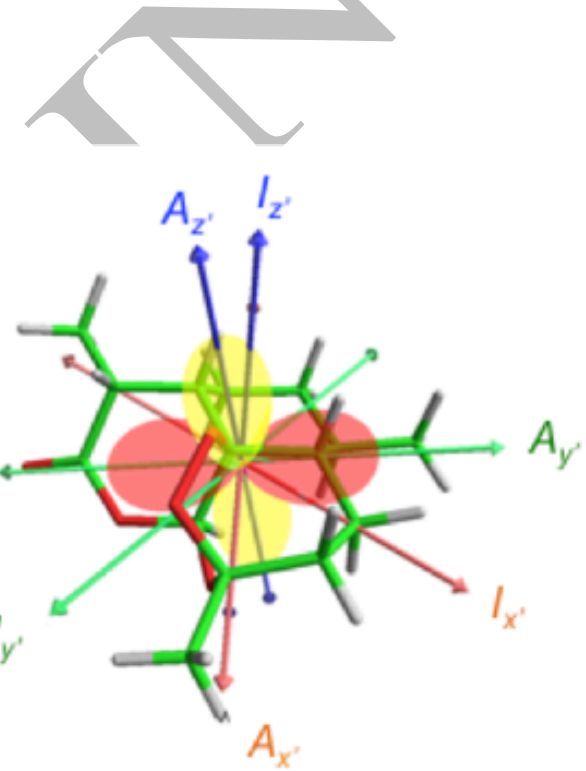

Artemisinin (2)

Figure 3. Displaying of principal axis system $\left(A_{\mathrm{x}^{\prime}}, A_{\mathrm{y}^{\prime}}, A_{\mathrm{z}}\right)$ of the diagonalized alignment tensor, the inertia tensor axes, $\left(I_{\mathrm{x}}, I_{\mathrm{y}^{\prime}}, I_{\mathrm{z}}\right)$ and the Saupe tensor surface representation (pink and yellow surfaces indicate positive and negative ${ }^{2} \mathrm{H}-\mathrm{RQCs}$, respectively) of $\mathbf{1}$ and $\mathbf{2}$ oriented in $\mathrm{PBLG} / \mathrm{CHCl}_{3}$.

Artemisinin. This is a very interesting tetracyclic compound due to its rare peroxide bridge, combined with seven stereogenic carbon positions and four prochiral $\mathrm{CH}_{2}$ groups (Figure 1). Artemisinin (2) was recently aligned in compressed PMMA/CDCl $\mathrm{P}_{3}$ gels and ${ }^{1} D_{\mathrm{CH}} \mathrm{RDCs}$ were reported. ${ }^{27}$ However, using only RDCs, a unique solution for the relative configuration problem was not found as two diastereoisomers showed similar quality factors, $Q .{ }^{27}$ In this second example, we reduced the amount of analyte to $50 \mathrm{mg}$, namely a molar concentration for each 
monodeuterated isotopomer of about $35 \mu \mathrm{mol} . \mathrm{I}^{-1}$. The analysis of the ANAD 2D-NMR spectrum of 2 recorded in PBLG/CHCl 3 leads to the unambiguous extraction of 15 signed RQCs out of 16 possible sites (Figure 2b and Table SI-4). As shown in Figure 1b, the correct absolute configuration of $\mathbf{2}$ is $1 S, 4 R, 5 S, 6 R, 7 S, 10 R, 11 R$, simplified here as SRSRSRR.

The same data analysis protocol used for strychnine was applied for artemisinin. The single tensor procedure was applied to handle those configurations presenting more than one conformation by fitting them in MSpin-RQC, along with alignment tensor components. ${ }^{70}$ Fitting the RQC data using the average values for the diastereotopic deuterons to all the possible relative configurations of $\mathbf{2}$, a lowest $Q$ factor of 0.064 was obtained for the relative configuration SRSRSRR (Table SI-6). The second-best score $(Q=0.103)$ was obtained for the relative configuration RRSRRSR, showing a clear discrimination of the correct structure.

Same as for the case of strychnine, the four methylenes of artemisinin lead to 16 possibilities for the assignment of the diastereotopic protons. Fitting all possible combinations of permuted assignment of diastereotopic deuterons leads to unambiguous assignment of the methylenic positions but one, the methylene at C-9. Sites $9 a$ and $9 b$ showed very similar ${ }^{2} \mathrm{H}-\mathrm{RQC}$ values of 436 and $-448 \mathrm{~Hz}$, and the $Q$ factors for the best fittings were 0.043 and 0.045 . H-9a and $\mathrm{H}-9 \mathrm{~b}$ can unambiguously assigned be based on the value of the vicinal coupling constants with H-10. Finally, all the above fitting procedures were repeated using the correct configuration structure (SRSRSRR) but with a geometry optimized by DFT. Using permutations, $Q$ factors of 0.017 and 0.018 (ambiguous solutions for $\mathrm{CH}_{2}-9$ ) were obtained, while a $Q=0.011$ was observed when the average RQCs for the diastereotopic site was used. In this case, significant differences in absolute $Q$ factors were observed between molecular mechanics and DFT-optimized structure geometries. Same as for $\mathbf{1}$, this quality of the fit is also confirmed by the excellent alignment of points on the ideal correlation plot (Figure SI-12), as well as the small relative average of R.D. values $(<4 \%)$. Table 1 lists all ordering-related parameters obtained from the experimental ${ }^{2} \mathrm{H}-\mathrm{RQCs}$ of $\mathbf{2}$. 
Table 1. Order-based molecular data extracted from ANAD 2D NMR

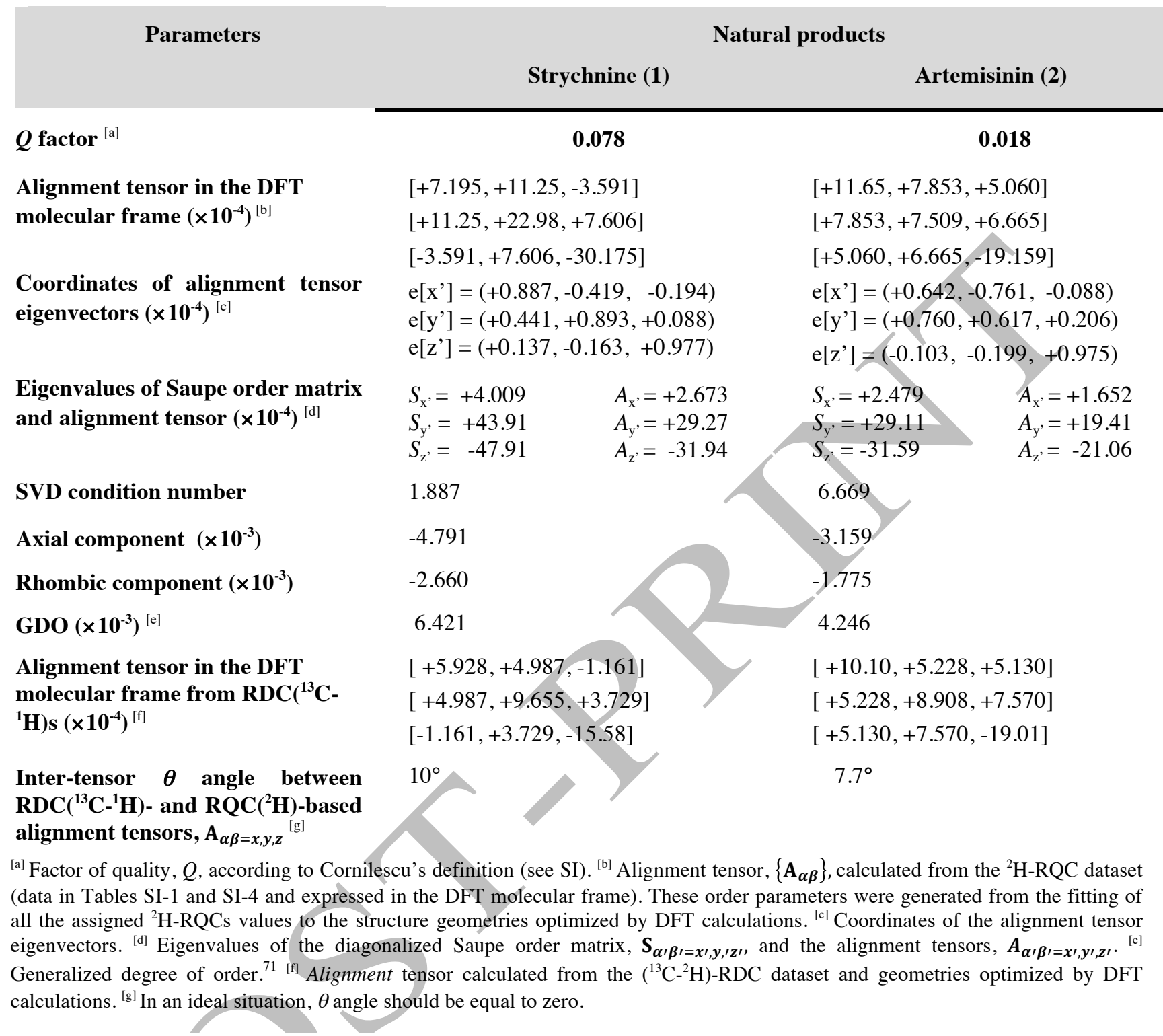

Comparison between the ${ }^{2} \mathrm{H}-\mathrm{RQC}$ - and $\left({ }^{13} \mathrm{C}-{ }^{1} \mathrm{H}\right)$-RDC-based Alignments. A validation of orientational ordering parameters obtained from experimental ${ }^{2} \mathrm{H}-\mathrm{RQCs}$ is possible by determining the inter-tensor angle between the alignment tensor, $A_{\alpha \beta=x, y, z}$, derived from ${ }^{2} \mathrm{H}-$ RQCs and $\left({ }^{13} \mathrm{C}-{ }^{1} \mathrm{H}\right)-\mathrm{RDCs}$. This $\theta$ angle was calculated from Eq. 1.

$$
\theta=\arccos \left(\frac{\sum_{\alpha \beta=x, y, z} A_{a \beta}^{\mathrm{RQC}} A_{a \beta}^{\mathrm{RDC}}}{\sqrt{\sum_{\alpha \beta=x, y, z}\left(A_{a \beta}^{\mathrm{RQC}}\right)^{2}} \sqrt{\sum_{\alpha \beta=x, y, z}\left(A_{a \beta}^{\mathrm{RDC}}\right)^{2}}}\right)
$$


In an ideal situation, the $\theta$ angle should be equal to zero. Here the small difference measured originates (Table 1) from the fact that experimental $\operatorname{RDC}\left({ }^{13} \mathrm{C}-{ }^{1} \mathrm{H}\right)$ listed in tables of Supporting Information are approximate values (not determined by CLIP-CLAP HSQC 2D spectra, for instance).

Correlation between Inertia and Alignment. To more deeply understand the alignment mechanisms of those solutes in the LLC phase, we displayed a representation in 3D space of the principal axes system for the alignment tensor, the inertia tensor as well as a valued-surface representation of the Saupe tensor. Figure $\mathbf{3}$ shows these graphical tools for compounds $\mathbf{1}$ and 2. It is worth noting the rather strong correlation between the eigenvectors of the inertia tensor of the diagonalized Saupe order matrix $\left(S_{x^{\prime}}, S_{y^{\prime}}, S_{z^{\prime}}\right)$, again showing the principally steric nature of molecular alignment of both analytes in the PBLG phases.

\section{CONCLUSION AND PROSPECTS}

Complementary to information provided by isotropic NMR, the nature of anisotropic NMR data provides, through molecular alignment, a direct spatial view of complex molecular structures as already shown in the case of $\left({ }^{13} \mathrm{C}-{ }^{1} \mathrm{H}-\right)-\mathrm{RDCs}$ and/or ${ }^{13} \mathrm{C}$-RCSA analysis. The use of natural abundance ${ }^{2} \mathrm{H}-\mathrm{RQCs}$ for the structural analysis of natural products is in a very young stage of its development, but no doubt it should be seen as a promising alternative to RDCs, or even as a change of paradigm in the domain of the elucidation of complex structures. Compared to anisotropic ${ }^{1} \mathrm{H}$ or ${ }^{13} \mathrm{C}$ NMR, the sensitivity of anisotropic NAD NMR is very low. However, considering the analytical benefits associated with the use of RQCs, this low sensitivity should not be considered as a prohibiting factor. The advent of very high magnetic field NMR instruments, the new generations of hardware and cryogenic probes, make possible the access to this new tool. This has been proven in this work where for the first time the relative configuration, including diastereotopic assignments, of complex natural products has been performed with the only use of NAD NMR. Although providing similar structural information to ${ }^{1} D_{\mathrm{CH}}$, the technique 
provides important advantages as NAD can be considered a pure-shift technique devoid of undesired strong coupling effects. As any new technology being developed, it is a matter of time to see anisotropic the NAD NMR approach available in a routine manner in the future, with the advent of innovative acquisition methods, ${ }^{76,77}$ or new chiral LLCs. ${ }^{78}$

There is no doubt that this new work paves the way for promising future studies. Thus, although doubling the experimental NMR time, the collection of ${ }^{2} \mathrm{H}-\mathrm{RQCs}$ at two different degrees of alignment by varying temperature or slightly diluting the sample, would also allow the determination of their signs. Around the same idea, the use of an algorithm able to explore all combinations of experimental data (RQC and sign) for a given ensemble of structures appears to be a possible strategy to simplify the analysis of data. A.I.-based programs could be a dramatic option. $^{79}$

Finally, as a new further frontier, NAD 2D NMR of small molecules self-aligned in strong magnetic fields, as demonstrated recently by Griesinger and co-workers, ${ }^{80}$ seems to be the next promising challenge.

\section{EXPERIMENTAL SECTION}

Oriented Samples. Analytes $\mathbf{1}\left(\mathrm{C}_{21} \mathrm{H}_{22} \mathrm{~N}_{2} \mathrm{O}_{2}, \mathrm{MW}=334.4 \mathrm{~g} / \mathrm{mol}\right)$ and $\mathbf{2}\left(\mathrm{C}_{15} \mathrm{H}_{22} \mathrm{O}_{5}\right.$, MW $=282.3$ $\mathrm{g} / \mathrm{mol}$ and the polypeptide (PBLG) were purchased from Sigma-Aldrich, and were used without further purification. The mole quantity of each analyte was $0.21 \mathrm{mmol}(70 \mathrm{mg}), 0.17 \mathrm{mmol}(50$ $\mathrm{mg}$ ), respectively. Chloroform-based polypeptide LLC samples (1 and 2) were prepared with 90 $\mathrm{mg}$ of PBLG and $600 \mathrm{mg}$ of $\mathrm{CHCl}_{3}$. The MW of PBLG was $162900 \mathrm{~g} / \mathrm{mol}(\mathrm{DP}=743)$. Here, the mass percentage of PBLG is about $12 \%$ for the two samples. From the point of view of ANAD NMR, polypeptide LLCs are well adapted for their ability to dissolve a large amount of high-MW analytes before losing their alignment properties.

Practical aspects for preparing oriented samples (fire-sealed, high-precision 5-mm NMR tubes) have been previously reported and will not be repeated here. ${ }^{41,46}$ As with any orienting 
environment, the minimization of orientational inhomogeneities of solute is crucial to ensure the highest possible resolution of anisotropic NAD spectra (FWHM $<3-5 \mathrm{~Hz}$ ), and to a maximized signal-to-noise ratio, SNR.

Note here that attempts to measure ${ }^{2} \mathrm{H}-\mathrm{RQCs}$ in compressed alignment gels compatible with chloroform (PMMA) and DMSO (poly-HEMA) ${ }^{72}$ failed due to strong polymer background signal of ${ }^{2} \mathrm{H}$ NMR at natural abundance, and limited volume inside the gel $(\sim 300 \mu \mathrm{L})$ to dissolve large amounts of analyte. E.g., Artemisinin precipitated inside the HEMA (DMSO) gel.

NMR Spectroscopy. Assignment of isotropic ${ }^{1} \mathrm{H}$ and ${ }^{13} \mathrm{C}$ resonances has been performed using classical ${ }^{1} \mathrm{H}-{ }^{1} \mathrm{H}$ COSY (@14.1 T) as well as ${ }^{13} \mathrm{C}-{ }^{1} \mathrm{H}$ HSQC and ${ }^{13} \mathrm{C}-{ }^{1} \mathrm{H} H M B C(@ 9.4 \mathrm{~T})$ 2D spectra in combination, when available, with data from the literature. ${ }^{41,65}$ Anisotropic NAD QUOSY 2D spectra (here the $Q$-resolved Fz 2D sequence) were recorded at $14.1 \mathrm{~T}(92.1 \mathrm{MHz}$ for ${ }^{2} \mathrm{H}$ ) on a Bruker Avance II spectrometer equipped with a 5-mm o.d. selective cryogenic probe. This pure-absorption in-phase 2D sequence allows refocusing of $\delta\left({ }^{2} \mathrm{H}\right) \mathrm{s}$ during the indirect dimension $t_{1}$, resulting in an advantageous reduction of the spectral window, $F_{1}$, compared to $F_{2}$ (and thus a decrease in the number of increments). ${ }^{39,51}$ All ${ }^{2} \mathrm{H}-{ }^{1} \mathrm{H}$ scalar and dipolar couplings were removed by WALTZ-16 CPD sequence. Sample temperature was controlled with a stability of $\pm 0.1 \mathrm{~K}$. The recycling delay and the number of points of the $2 \mathrm{D}$ matrix $\left(t_{2}, t_{1}\right)$ and scans $(<200)$ per $t_{1}$ increments of $Q$-resolved Fz 2D experiments (values are given in the Supporting Information) were optimized to record spectra within 20-23 hours using the $2 \mathrm{D}$. Adapted exponential filtering $\left(\mathrm{LB}_{1,2}=4\right.$ to $\left.8 \mathrm{~Hz}\right)$ and zero-filling to $4 \mathrm{k}\left(t_{2}\right) \times 4 \mathrm{k}\left(t_{1}\right)$ were applied prior to the two-dimensional Fourier transform. When necessary, further experimental details are given in the figure captions. ${ }^{1} D_{\mathrm{C}-\mathrm{H}}$ values listed in Tables of the Supporting Information were determined as the difference ${ }^{1} D_{\mathrm{CH}}=\left({ }^{1} T_{\mathrm{CH}}-{ }^{1} J_{\mathrm{CH}}\right) / 2 .{ }^{1} T_{\mathrm{CH}}$ and ${ }^{1} J_{\mathrm{CH}}$ values were extracted from the anisotropic and isotropic proton-coupled ${ }^{13} \mathrm{C} 1 \mathrm{D}$ NMR and $J / T$-resolved 2D spectra, respectively. 
Computational Methodology. All possible relative configurations for chiral substances $\mathbf{1}$ and 2 were generated using the LigPrep program (Schrödinger, LLC). For each configuration a conformational search was performed at the MMFF94 level using the Monte Carlo multiple minimum algorithm as implemented in the Macro Model program (Schrödinger, LLC). ${ }^{73}$ EFG tensors were computed by single point B3LYP/6-31G* computations on the MMFF94 structures. Alignment tensors were then computed for each conformational ensemble using the MSpin-RQC program as previously described. ${ }^{35,53}$ For those configurations having more than one populated conformation the single tensor approximation. ${ }^{74,75}$ was employed. EFG tensors were Boltzmann averaged by using the computed B3LYP SCF energies at a temperature of $298.15 \mathrm{~K}$.

\section{AUTHORS INFORMATION}

Notes. The authors declare no competing financial interest.

\section{ACKNOWDEGMENTS}

P.L. and P.B. acknowledge both the CNRS and the Université Paris-Saclay (previously Université Paris-Sud) for their support and recurrent funding of fundamental research. A.N.-V. thanks FACEPE for financial support (APQ).

\section{REFERENCES}

(1) Cid, M. in Structure Elucidation in Organic Chemistry. J. Wiley \& Sons, 2015.

(2) Liu, Y.; Sauri, J., Mevers E.; Peczuh, M. W.; Hiemstra, H.; Clardy J. Martin, G. E. Unequivocal determination of complex molecular structures using anisotropic NMR measurements. Science 2017, 356, eaam5349.

(3) Wu, J.; Lorenzo, P., Zhon, S.; Ali, M.; Butts C. P.; Myers, E. L.; Aggarwal, V. K. Synergy of synthesis, computation and NMR reveals correct baulamycin structures. Nature 2017, $547,436-440$.

(4) Williams, A. J.; Martin G. E.; Rovnyak, D.; New directions in natural products NMR: What can we learn by examining how the discipline has Evolved? Chap. 1, pp 1-25, in Modern NMR Approaches to the Structure Elucidation of Natural Products, Vol. 1 Instrumentation and Software, (Eds Williams, A. J.; Martin, G. E.; Rovnyak, D. RSC, Cambridge, 2015. 
(5) Li. G.-W.; Liu, H.; Qiu, F.; Wang, X.-J.; Lei, X.-X. Residual dipolar couplings in structure determination of natural products, Natural Products and Bioprospecting. 2018, 8, 279-295.

(6) Carlomagno, T. NMR in natural products: understanding conformation, configuration and receptor interactions. Nat. Prod. Rep. 2012, 29, 536-554.

(7) Wunderlich, C. H.; Grutsch, S.; Tollinger, M.; Kreutz, C. Nuclear Magnetic Resonance of Large Molecules in Natural Products. 253, 1-18, John Wiley \& Sons, Ltd, 2006.

(8) Karki, S.; Fábián, L.; Friscić, T.; Jones, W. Powder X-ray diffraction as an emerging method to structurally characterize organic solids. Org. Lett. 2007, 9, 3133-3136.

(9) Warren, M. Why didn't we think to do this earlier? Chemists thrilled by speedy atomic structures. Nature 2018, 563, 16-17.

(10) Inokuma, Y.; Inokuma, Y.; Yoshioka, S.; Ariyoshi, J.; Arai, T.; Hitora, Y.; Takada, K.; Matsunaga, S.; Rissanen, K.; Fujita, M. X-ray Analysis on the nanogram to microgram scale using porous complexes. Nature 2013, 495, 461-466.

(11) Reynolds, W.F. Natural product structure elucidation by NMR spectroscopy, pp. 567597, in Pharmacognosy: Fundamentals, Applications and Strategies, Badal, S. and Delgoda R. (Eds.) Academic Press-Elsevier Ltd Science Ltd, London.

(12) Neuhaus, D.; Williamson, M. P. in The Nuclear Overhauser Effect in Structural and Conformational Analysis Wiley-VCH, Wiley-VCH, 2000.

(13) Gil, R. R.; Navarro-Vázquez, A. in Modern NMR Approaches to the Structure Elucidation of Natural Products: Volume 2: Data Acquisition and Applications to Compound Classes 2, 1-38; RSC, 2016.

(14) Altona, C. in Encyclopedia of NMR, pp 4909-4923, Eds. D.M. Grant and R. Morris, 1996, New York.

(15) Matsumori, N.; Kaneno, D.; Murata, M.; Nakamura, H.; Tachibana, K. Stereochemical determination of acyclic structures based on carbon-proton spin-coupling constants. A method of configuration analysis for natural products. 1999, 64, 866-876.

(16) Butts, C. P.; Jones, C. R.; Harvey, J. N. High precision NOEs as a probe for low level conformers-a second conformation of strychnine. Chem. Commun. 2011, 47, 11931195.

(17) Bifulco, G.; Dambruoso, P.; Gomez-Paloma, L.; Riccio, R. Determination of relative configuration in organic compounds by NMR spectroscopy and computational methods. Chem. Rev. 2007, 107, 3744-3779.

(18) Grimblat, N.; Sarotti, A. M. Computational chemistry to the rescue: Modern toolboxes for the assignment of complex molecules by GIAO NMR calculations. Chem. Eur. J. 2016, 22, 12246-12261.

(19) Kutateladze, A. G.; Mukhina, O. A. Relativistic force field: Parametric computations of 
proton-proton coupling constants in ${ }^{1} \mathrm{H}$ NMR spectra. J. Org. Chem. 2014, 79, 83978406.

(20) Gil, R. R. in Encyclopedia of Spectroscopy and Spectrometry 946-955 (Elsevier, 2017). doi:10.1016/B978-0-12-409547-2.12127-4.

(21) Schuetz, A.; Junker, J.; Leonov, A.; Lange, O. F.; Molinski, T. F.; Griesinger C. Stereochemistry of sagittamide A from residual dipolar coupling enhanced NMR. J. Am. Chem. Soc. 2007, 129, 15114-15115.

(22) Sun, H.; Reinscheid, U. M.; Whitson, E. L.; d'Auvergne, E. J., Ireland, C. M.; NavarroVazquez, A.; Griesinger, C. Challenge of large-scale motion for residual dipolar coupling based analysis of configuration: the case of fibrosterol sulfate A. J. Am. Chem. Soc. 2011, $133,14629-14636$.

(23) Verdier, L.; Sakhaii, P.; Zweckstetter, M.; Griesinger, C. Measurement of long range $\mathrm{H}, \mathrm{C}$ couplings in natural products in orienting media: a tool for structure elucidation of natural products. J. Magn. Reson. 2003, 163, 353-359.

(24) Nath, N.; d'Auvergne; E. J.; Griesinger, C. Long-range residual dipolar couplings: A tool for determining the configuration of small molecules. Angew. Chem. Int. Ed. 2015, 54, 12706-12710.

(25) Waratchareeyakul, W.; Helleman, E.; Gil. R. R.; Chantrapromma, K.; Langat, M. K.; Mulholland D. A. Application of Residual dipolar couplings and selective quantitative NOE to establish the structures of tetranortriterpenoids from Xylocarpus rumphii. J. Nat. Prod. 2017, 80, 391-402.

(26) Gil, R. R.; Gayathri, C.; Tsarevsky, N. V.; Matyjaszewski, K. Stretched poly(methyl methacrylate) gel aligns small organic molecules in chloroform. stereochemical analysis and diastereotopic proton NMR assignment in ludartin using residual dipolar couplings and ${ }^{3} \mathrm{~J}$ coupling constant analysis. 2008, 73, 840-848.

(27) Navarro-Vázquez, A.; Gil, R. R.; Blinov, K. Computer-Assisted 3D structure elucidation (CASE-3D) of natural products combining isotropic and anisotropic NMR parameters. $J$. Nat. Prod. 2018, 81, 203-210.

(28) Hallwass, F.; Schmidt, S.; Sun. H. Mazur A. Kummerlöwe, G. Luy, B.; Griesinger, C.; Reinscheid, U. M. Residual chemical shift anisotropy (RCSA): a tool for the analysis of the configuration of small molecules. Angew. Chem. Int. Ed. 2011, 50, 9487-9490.

(29) Kummerlöwe, G.; Grage S. L.; Thiele, C. M.; Kuprov, I.; Ulrich; S. A.; Luy, B. Variable angle NMR spectroscopy and its application to the measurement of residual chemical shift anisotropy. J. Magn. Reson. 2011, 209, 19-30.

(30) Nath, N.; Schmidt; M.; Gil, R.R.; Willamson; R.T.; Martin, G. E.; Navarro-Vazquez, A.; Griensinger, C.; Liu Y. Determination of relative configuration from residual chemical shift anisotropy. J. Am. Chem. Soc. 2016, 138, 9548-9556.

(31) Mevers, E.; Saurí, J.; Liu, Y.; M. A., Ramadhar T.M.; Varlan, M.; Williamson, R.T.; Martin, G.E.; Clardy, J. Homodimericin A: A complex hexacyclic fungal metabolite. $J$. Am. Chem. Soc. 2016, 138, 12324-12327. 
(32) Gil, R. R.; Hellemann, E.; New stretching method for aligning gels. Its application to the measurement residual chemical shift anisotropies (RCSAs) without the need for isotropic shift correction. Chem. Eur. J. 2018, 24, 3689-3693.

(33) Hallwass, F.; Teles, R. R.; Hellemann, E.; Griesinger, C.; Gil, R. R.; Navarro-Vázquez, A. Measurement of residual chemical shift anisotropies in compressed polymethylmethacrylate gels. Automatic compensation of gel isotropic shift contribution. Magn. Reson. Chem. 2018, 56, 321-328.

(34) Liu, Y.; Navarro-Vázquez, A.; Gil. R. R.; Griesinger C.; Martin G. E.; Williamson, R.T. Application of anisotropic NMR parameters to the confirmation of molecular structure, Nature Protocols. 2019, 14, 217-247.

(35) Navarro-Vázquez, A.; Berdagué, P.; Lesot, P. Integrated computational protocol for the analysis of quadrupolar splittings from natural-abundance deuterium NMR spectra in (chiral) oriented media. ChemPhysChem. 2017, 18, 1252-1266.

(36) Sarfati, M.; Lesot, P.; Merlet, D.; Courtieu. J. Theoretical and experimental aspects of enantiomeric differentiation using natural abundance multinuclear NMR spectroscopy in chiral polypeptide liquid crystals. Chem. Commun. 2000, 2069-2081.

(37) Lesot, P.; Courtieu, J. Natural abundance deuterium NMR spectroscopy: Developments and analytical applications in liquids, liquid crystals and solid phases. Prog. Nucl. Magn. Reson. Spectrosc. 2009, 55, 128-159.

(38) Lesot, P. Deuterium NMR of liquid-crystalline samples at natural abundance. eMagRes. 2013, 315-334, J. Wiley \& Sons, doi:10.1002/9780470034590.emrstm1318.

(39) Lesot, P.; Aroulanda C.; Berdagué, P.; Meddour, A. Merlet, D.; Farjon, J.; Giraud, N.; Lafon O. Multinuclear NMR in polypeptide liquid crystals: Three fertile decades of methodological developments and analytical challenges, Prog. Nucl. Magn. Reson. Spectrosc. 2020, 116, 85-154.

(40) Lesot, P.; Merlet, D.; Loewenstein, A.; Courtieu, J. Enantiomeric visualization using proton-decoupled natural abundance deuterium NMR in poly $(\gamma$-benzyl- $L$-glutamate $)$ liquid crystalline solutions. Tetrahedron: Asymmetry. 1998, 9, 1871-1881.

(41) Lesot, P.; Sarfati, M.; Courtieu, J. Natural abundance deuterium NMR spectroscopy in polypeptide liquid crystals as a new and incisive means for the enantiodifferentiation of chiral hydrocarbons. Chem. Eur. J. 2003, 9, 1724-1745.

(42) Kovacs, H.; Moskau, D.; Spraul, M. Cryogenically cooled probes-a leap in NMR technology. Prog. Nucl. Magn. Reson. Spectrosc. 2005, 46, 131-155.

(43) Anklin, C. in Modern NMR Approaches to the Structure Elucidation of Natural Products: Volume 1: Instrumentation and Software 1, 38-57 (Royal Society of Chemistry, 2015).

(44) Ndukwe, I.E.; Ndukwe, I.E.; Wang, X.; Pelczer, I.; Reibarkh. M.; Williamson, R.T.; Liu. 
Y.; Martin G.E. PBLG as a versatile liquid-crystalline medium for anisotropic NMR data acquisition. Chem. Commun., 2019, 55, 4327-4330.

(45) Lesot, P., Determination of the natural deuterium distribution of fatty acids by application of ${ }^{2} \mathrm{H}$ 2D-NMR in liquid crystals: fundamentals, advances, around and beyond, Liq. Crystals, Published online: https://doi.org/10.1080/02678292.2019.1613685.

(46) Berdagué, P.; Lesot, P.; Jacob, J.; Terwilliger, V .J.; Le Milbeau, C. Contribution of NAD 2D-NMR in liquid crystals to the determination of hydrogen isotope profile of methyl groups in miliacin. Geochimica et Cosmochimica Acta. 2016, 173, 337-351.

(47) Texier-Bonniot, T.; Berdagué, P.; Robins, R.; Remaud, G.; Lesot, P. Analytical contribution of deuterium 2D-NMR in oriented solvents to ${ }^{2} \mathrm{H} /{ }^{1} \mathrm{H}$ isotopic characterization: the case of vanillin Flav. Frag. Journal 2018, 34, 217-229.

(48) Edgar, M.; Emsley, J. W.; Furby, M. I. C. General features of the X part of an ABX spin system in isotropic and liquid crystalline phases as illustrated by the ${ }^{13} \mathrm{C}-\left\{{ }^{1} \mathrm{H}\right\}$ spectra of 2,2'-difluorobiphenyl. J. Magn. Reson. 1997, 128, 105-113.

(49) Yu. B.; van Ingen, H.; Vivekanandan, S.; Rademacher, C.; Norris, S. E. ; Freedberg. D.I. More accurate ${ }^{1} \mathrm{~J}_{\mathrm{CH}}$ coupling measurement in the presence of ${ }^{3} \mathrm{~J}_{\mathrm{HH}}$ strong coupling in natural abundance. J. Magn. Reson. 2012, 215, 10-22.

(50) Merlet, D.; Ancian, B.; Courtieu, J.; Lesot, P. Two-Dimensional deuterium NMR spectroscopy of chiral molecules oriented in a polypeptide liquid crystal: Applications for the enantiomeric analysis through natural abundance deuterium NMR. J. Am. Chem. Soc. 1999, $121,5249-5258$.

(51) Lafon, O.; Lesot, P.; Merlet, D.; Courtieu, J. Modified z-gradient filtering as a mean to obtain phased deuterium autocorrelation 2D NMR spectra in oriented solvents. J. Magn. Reson. 2004, 171, 135-142.

(52) Lesot, P.; Lafon, O. Enantiomeric analysis using natural abundance deuterium 3D NMR spectroscopy in polypeptide chiral oriented media. Chem. Phys. Lett. 2008, 458, 219222.

(53) Navarro-Vázquez, A. MSpin-RDC. A program for the use of residual dipolar couplings for structure elucidation of small molecules. Magn. Reson. Chem. 2012, 50, S73-S79.

(54) Troche-Pesqueira, E.; Anklin, C.; Gil, R. R.; Navarro-Vázquez, A. Computer-assisted 3D structure elucidation of natural products using residual dipolar couplings. Angew. Chem. Int. Ed. 2017, 56, 3660-3664.

(55) Willoughby, P.; Jansma, M. J.; Hoye, T. R. A guide to small-molecule structure assignment through computation of $\left({ }^{1} \mathrm{H}\right.$ and $\left.{ }^{13} \mathrm{C}\right)$ NMR chemical shifts. Nature Protocols 2014, 9, 643-660.

(56) Carter, J. C.; Luther, G. W.; Long, T. C. Proton magnetic resonance spectra and assignments of strychnine and selectively deuterated strychnine. J. Magn. Reson. 1974, 
$15,122-131$.

(57) Bagno, A.; Rastrelli, F.; Saielli, G. Toward the complete prediction of the ${ }^{1} \mathrm{H}$ and ${ }^{13} \mathrm{C}$ NMR spectra of complex organic molecules by DFT methods: Application to natural substances. Chem. Eur. J. 2006, 12, 5514-5525.

(58) Thiele, C. M.; Berger, S. Probing the Diastereotopicity of methylene protons in strychnine using residual dipolar couplings. Org. Lett. 2003, 5, 705-708.

(59) Snider, J. D.; Trocje-Perquiera, E.; Woodruff, S. R.; Gayathri C.; Tsatevsky N. V.; Gil, R. R. New strategy for RDC-assisted diastereotopic proton assignment using a combination of J-scaled BIRD HSQC and J-scaled BIRD HMQC/HSQC. Magn. Reson. Chem. 2012, 50, S86-S91.

(60) Schmidt, M.; Sun, H.; Leonov, A.; Griesinger, C.; Reinscheid, U. M. Chiral discrimination of amines by anisotropic NMR parameters using chiral polyacrylamidebased gels. Magn. Reson. Chem. 2012, 50, S38-S44.

(61) Bifulco, G.; Riccio, R.; Martin, G. E.; Buevich, A. V.; Williamson, R. T. Quantum chemical calculations of ${ }^{1} \mathrm{~J}(\mathrm{CC})$ coupling constants for the stereochemical determination of organic compounds. Org. Lett. 2013, 15, 654-657.

(62) Kolmer, A.; Edwards, L. J.; Kuprov, I.; Thiele, C. M. Conformational analysis of small organic molecules using NOE and RDC data: A discussion of strychnine and $\alpha$ methylene- $\gamma$-butyrolactone. J. Magn. Reson. 2015, 261, 101-109.

(63) Butler, A. R.; Conforti, L.; Hulme, P.; Renton, L. M.; Rutherford, T. J. NMR and conformational studies of the antimalarial drug arte-ether. Journal of the Chemical Society, Perkin Transactions 2, 1999, 10, 2089-2092.

(64) El-feraly, F. S.; El-sherei, M. M.; Hufford, C. D.; Croom, E. M., Jr; Mahier, T. J. ${ }^{13} \mathrm{C}$ NMR Assignments of artemisinin, desoxyartemisinin and artemether. Spectroscopy Lett. 2006, 18, 843-849.

(65)Blaskó, G.; Cordell, G. A.; Lankin, D. C. Definitive ${ }^{1} \mathrm{H}-$ and ${ }^{13} \mathrm{C}-\mathrm{NMR}$ assignments of artemisinin (Qinghaosu). J. Nat. Prod. 2004, 51, 1273-1276.

(66) Dembitsky, V.M. Astonishing diversity of natural peroxides as potential therapeutic agent, J. Mol. Genet. Med. 2015, 9, 1 -18.

(67) Aroulanda, C.; Lesot, P.; Merlet, D.; Courtieu, J. Structural ambiguities revisited in two bridged ring systems exhibiting enantiotopic elements, using natural abundance deuterium NMR in chiral liquid crystals. J. Phys. Chem. A 2003, 107, 10911-10918.

(68) Cobas, J. C.; Constantino Castillo, V.; Martín Pastor, M.; del Río Portilla, F. A two-stage approach to automatic determination of ${ }^{1} \mathrm{H}$ NMR coupling constants. Magn. Reson. Chem. 2005, 43, 843-848.

(69) Thiele, C. M. Simultaneous assignment of all diastereotopic protons in strychnine using RDCs: PELG as alignment medium for organic molecules. J. Org. Chem. 2004, 69, 7403-7413. 
(70) Smith, G.; Wermuth, U. D.; Healy, C. P.; Young D. J., Strychnine-8-ammonio-2 naphthalene-sulfonate-water (1/1/3.5): The first structure of a strychnine or brucine compound with a zwitterionic species. J. Chem. Cryst. 2006, 6, 805-811.

(71) Kramer, F.; Deshmukh, M.V.; Kessler H.; Glaser, S.J. Residual dipolar coupling constants: An elementary derivation of key equations, Concepts Magn. Reson. A. 2004, $21,10-21$.

(72) Gil-Silva, L. F.; Santamaría-Fernández, R.; Navarro-Vázquez, A.; Gil, R. R. Collection of NMR scalar and residual dipolar couplings using a single experiment. Chem. Eur. $J$. 2015, 22, 472-476.

(73) LigPrep and MacroModel; Schrodinger LLC: New-York, 2020. https://www .schrodinger.com.

(74) Thiele, C. M.; Schmidts, V.; Böttcher, B.; Louzao, I.; Berger, R.; Maliniak, A.; Stevensson, B. On the treatment of conformational flexibility when using residual dipolar couplings for structure determination. Angew. Chem. Int. Ed. 2009, 48, 6708-6712.

(75) Burnell, E. E.; De Lange, C. A. Effects of interaction between molecular internal motion and reorientation on NMR of anisotropic liquids. J. Magn. Reson. 1980, 39, 461-480.

(76) Lafon, O.; Hu, B.; Amoureux J.-P.; Lesot P. Fast and high-resolution stereochemical analysis by non-uniform sampling and covariance processing of anisotropic natural abundance 2D ${ }^{2} \mathrm{H}$ NMR datasets Chem. Eur. J. 2011, 17, 6716-6724.

(77) Kazimierczuk, K.; Lafon, O.; Lesot, P. Criteria for sensitivity enhancement by compressed sensing: practical application to anisotropic NAD 2D-NMR spectroscopy, Analyst, 2014, 139, 2702-2713.

(78) Lesot, P.; Berdagué P.; Meddour A.; Kreiter A., Noll, M.; Reggelin, M. ${ }^{2} \mathrm{H}$ and ${ }^{13} \mathrm{C}$ NMR-based enantiodetection using polyacetylene versus polypeptide aligning media: versatile and complementary tools for chemists. ChemPlusChem. 2019, 84, 144-153.

(79) Burnell, E. E.; de Lang, C. A.; Dong R. Y.; Leo Meerts W.; Weber, A. C. J. Evolutionary algorithms and nuclear magnetic resonance of oriented molecules Concepts Magn. Reson., Part A. 2016, 45A:e21415. pp. 1-14.

(80) Karschin, N.; Wolkenstein K.; Griesinger, C. Magnetically induced alignment of natural products for stereochemical structure determination via NMR. Angew. Chem. Int. Ed. 2020. 10.1002/anie. 202004881. 